\title{
A Fair Downlink Scheduling Algorithm for 3GPP LTE Networks
}

\author{
S. M. Chadchan \\ B.L.D.E.A's V. P. Dr. P. G. H. College of Engineering and Technology, Bijapur, India \\ sanjeevchadchan@yahoo.com \\ C. B. Akki \\ Wipro Technologies, Bangalore, India \\ channappa.akki@wipro.com
}

\begin{abstract}
Long Term Evolution (LTE), the standard specified by 3GPP on the way towards the fourth generation mobile networks, is intended to deliver high speed data and multimedia services to next generation mobile users. The Radio Resource Management (RRM) plays a very crucial role in managing limited radio resources to enhance system's data rate and to ensure quality of service (QoS) provisioning in both uplink and downlink. LTE downlink scheduling is a RRM entity that is responsible for the allocation of shared radio resources among mobile user equipment (UEs). One of the challenges that need to be addressed in downlink scheduling algorithms is to provide higher spectral efficiency with fairness ensured to all its users. This paper proposes a novel downlink scheduling algorithm which balances its performance between efficiency (in terms of throughput) and fairness to users. The algorithm makes use of Assignment Model for resource allocation to all the selected users during each Transmission Time Interval (TTI). The proposed algorithm is simulated and the simulation results are compared with three techniques proposed in literature. The simulator results show that the new algorithm balances well between throughput and fairness.
\end{abstract}

Index Terms - OFDM, QoS, Resource Blocks, CQI, TTI

\section{INTRODUCTION}

The Third Generation Partnership Project (3GPP) has standardized Long Term Evolution (LTE) / System Architecture Evolution (SAE) in Release- 8 to build the framework for $3 \mathrm{G}$ evolution towards $4 \mathrm{G}$. The motivation for $3 \mathrm{G}$ evolution came from the growing demand for network services such as VoIP, web browsing, video telephony, and video streaming, with constraints on delays and bandwidth requirements. LTE aims to support all the applications with better performance at reduced cost, besides maintaining seamless mobility [1-6]. LTE is an all-IP packet based system with design targets of supporting high peak data rates of $100 \mathrm{Mb} / \mathrm{s}$ in the downlink (DL) and $50 \mathrm{Mb} / \mathrm{s}$ in the uplink (UL), low latency (10ms round-trip delay), improved system capacity and coverage $[7,8]$. To achieve the performance objectives, LTE employs the several enabling technologies which include Orthogonal Frequency Division Multiple Access (OFDMA) [9], Single Carrier Frequency Division Multiple Access (SC-FDMA) [10] and Multiple Input Multiple Output (MIMO) [11]. LTE technology uses air interface based on OFDMA for downlink and SC-FDMA for uplink. The LTE operates in both Time Division Duplexing (TDD) and Frequency Division Duplexing (FDD) modes and can be deployed in a range of channel bandwidths up to $20 \mathrm{MHz}$.

The QoS provisioning has been an important issue in the mobility management of wireless networks [12-17]. To provide QoS in wireless networks, the Radio resource management (RRM) plays a crucial role in managing the limited radio resources effectively [18]. The Packet Scheduler is an entity of RRM in LTE that resides in the MAC layer of eNodeB [19-22]. It is responsible for the allocation of shared radio resources among the mobile User Equipments (UEs). The scheduler assigns resources to users with the granularity of resource blocks (RBs) every TTI, based on the channel condition feedback received from UEs in the form of Channel Quality Indicator (CQI).

This paper focuses on Downlink scheduling in LTE. Several scheduling strategies have been proposed in the literature [23-35]. In Round Robin scheduling algorithm, the UEs are assigned the resources in round-robin fashion, without taking into account channel conditions. The Max $\mathrm{C} / \mathrm{I}$ (or Best CQI) algorithm allocates the resource blocks to the UEs with highest CQI on RB during a TTI [1]. Round-Robin algorithm offers fairness with respect to time to all UEs, but it is less efficient with respect to throughput because it doesn't take into account channel variations. The Best CQI algorithm is efficient, but it is not fair to all users. The UEs, such as those at the cell edges, which face bad channel conditions, will always not get RBs allocated. Hence such users always starve of radio resources, which is practically not acceptable. So, fairness should also be taken into account along with focus on spectral efficiency. In [33], the proposal for the fair scheduling algorithms is made, in which RB allocation is done to all chosen UEs in a TTI, selecting 
UEs one after another starting from the one with highest $\mathrm{CQI}$ onwards in order. Once a UE gets $\mathrm{RB}$, it is not assigned further till other users are assigned $\mathrm{RBs}$ or till the RBs are free within a TTI. This paper proposes a novel scheduling algorithm based on the one proposed

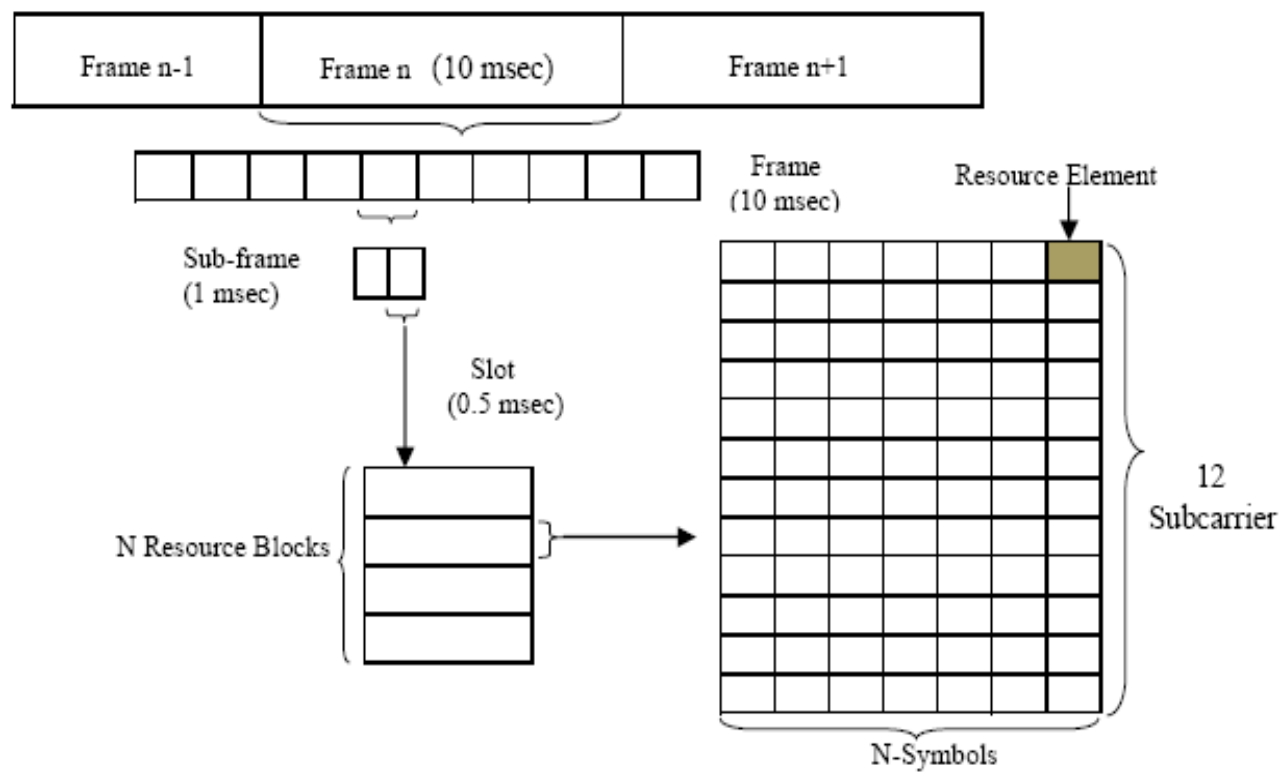

Figure 1: LTE air interface elements.

in [33] and explores the possibility of improving overall throughput of the cell, besides maintaining fairness to users. The performance of the algorithm is investigated using a MATLAB based LTE link level simulator from Vienna University [36].

The rest of the paper is organized as follows. The section II deals with the framework for LTE Downlink scheduling. The section III discusses the proposed algorithm for LTE Downlink scheduling. The simulation results are discussed in section IV. Finally, section V concludes the paper.

\section{LTE DOWNLINK SCHEDULING}

\section{A. LTE Downlink Air Interface}

The LTE downlink air-interface employs Orthogonal Frequency Division Multiplexing (OFDM) together with advanced antenna techniques and adaptive modulation and coding (AMC) to achieve better spectral efficiency, which enables operators to transfer more data per $\mathrm{MHz}$ of spectrum at lower cost-per-bit. OFDM is a multicarrier transmission scheme, in which the available bandwidth is divided into multiple narrow sub-carriers and data is transmitted on these sub-carriers in parallel streams. Each sub-carrier is then modulated using different levels of modulation such as QPSK, 16-QAM and 64-QAM. OFDM has the benefits of robustness against frequency selective fading, superior spectral flexibility, bandwidth scaling, and efficient receiver architecture.

The resource allocation in LTE is done in time/frequency domain. The LTE air interface elements are shown in Fig. 1. In time-domain, the DL channels in air interface are divided into Frames of $10 \mathrm{~ms}$ each. A frame consists of ten Subframes, each of $1 \mathrm{~ms}$. Each subframe interval is referred to as Transmission Time Interval (TTI). Each subframe consists of two Slots of $0.5 \mathrm{~ms}$. In frequency domain, the total available system bandwidth is divided into sub-channels of $180 \mathrm{kHz}$, with each sub-channel comprising of 12 consecutive equally spaced OFDM sub-carriers of $15 \mathrm{KHz}$ each. A timefrequency radio resource spanning over $0.5 \mathrm{~ms}$ slots in the time domain and over $180 \mathrm{KHz}$ sub-channel in the frequency domain is called Resource Block (RB). Each $\mathrm{RB}$ carries OFDM symbols and the number of symbols per RB depends upon cyclic prefix being used (typically 6 or 7). The number of resource blocks in the available bandwidth is called Resource grid. Resource Element (RE) represents one OFDM subcarrier during one OFDM symbol interval. The number of RBs in a resource grid depends on the size of the bandwidth. The LTE operates in the bandwidth of $1.4 \mathrm{MHz}$ up to $20 \mathrm{MHz}$, with number of RBs ranging from 6 to 100 for bandwidths $1.4 \mathrm{MHz}$ to $20 \mathrm{MHz}$ respectively, as shown in Table I [37].

Table I. LTE Bandwidth and RB Specifications

\begin{tabular}{|l|l|l|l|l|l|l|}
\hline Bandwidth(MHz) & 1.4 & 3 & 5 & 10 & 15 & 20 \\
\hline Number of RBs & 6 & 15 & 25 & 50 & 75 & 100 \\
\hline $\begin{array}{l}\text { Number of sub- } \\
\text { carriers occupied }\end{array}$ & 72 & 180 & 300 & 600 & 900 & 1200 \\
\hline
\end{tabular}

\section{B. LTE Downlink Scheduling Framework}

Multi-user Packet-scheduling plays a vital role in the distribution of available radio resources among the active users in order to ensure QoS needs of users. In order to counteract the time-varying and frequency-selective nature of the wireless channel, the scheduler needs to allocate resources (on per RB basis) to appropriate UEs 
which have good channel conditions based on the selected metric, every TTI. The metric is used as transmission priority of each $\mathrm{UE}$ on each $\mathrm{RB}$, and is defined based on channel quality, status of transmission queues, buffer state, QoS requirements and resource allocation history. Packet schedulers are deployed at eNodeB and they allocate resources with a granularity of one TTI and one RB in time and frequency domain respectively. In order to perform channel-aware packet scheduling, each eNodeB need to have the knowledge of Channel State Information (CSI) for each user, for all the RBs in the available bandwidth. The CSIs are derived based on channel gain, interference conditions and Signal to interference noise ratio (SINR) estimation errors.

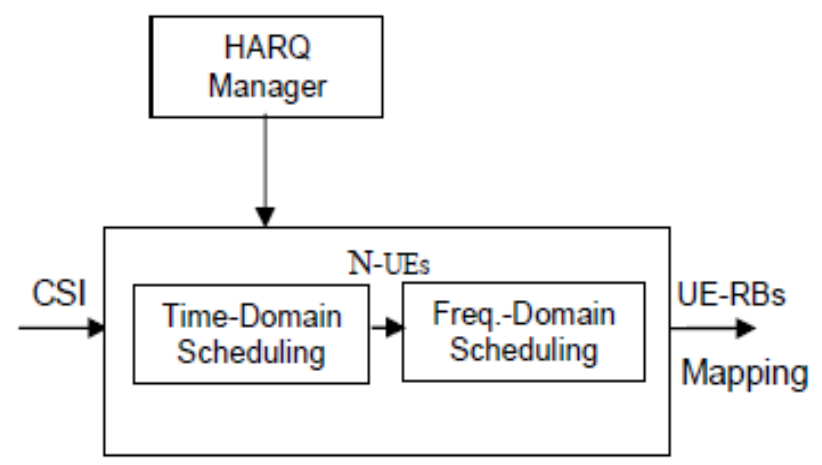

Figure 2: LTE Packet scheduler framework in RRM

The LTE packet scheduling task comprises of two subtasks: Time-domain packet scheduling (TDPS) and Frequency-domain packet scheduling (FDPS) as shown in the Fig. 2 [30]. TDPS identifies UEs that can transmit in the next TTI, taking into considerations input from HARQ manager, which provide the set of UEs that need to retransmit in a specific TTI. In order to improve the spectral efficiency of the system, FDPS tries to allocate each RB to appropriate UE such that the value of $\mathrm{M}_{\text {sum }}$ is maximized in eqn. (1).

$M_{\text {sum }}=\sum M(i, j) x A(i, j)$

where $\mathrm{M}(\mathrm{i}, \mathrm{j})$ is the channel aware metric of $i^{\text {th }} \mathrm{UE}$ for $j^{\text {th }}$ RB. $\mathrm{A}(\mathrm{i}, \mathrm{j})=1$ or 0 , if $i^{\text {th }} \mathrm{UE}$ is allocated $j^{\text {th }} \mathrm{RB}$ or otherwise respectively. The channel aware metric values $\mathrm{M}(\mathrm{i}, \mathrm{j})$ for different users on different RBs is shown in Fig. 3 [30].

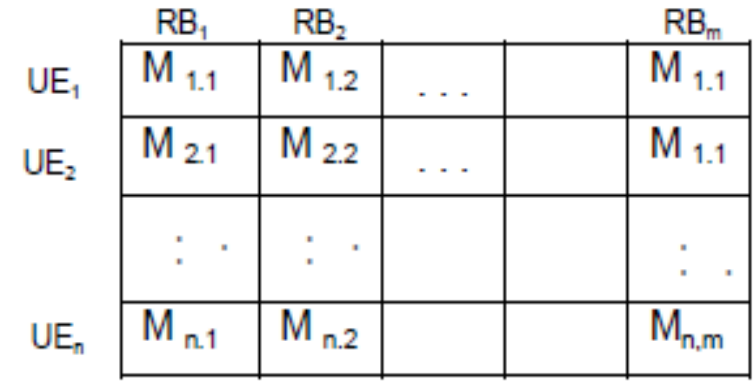

Figure 3: Metric values for each user and each RB

The design of LTE downlink scheduler is a complex process and it poses a number of challenges such as maximization of system capacity, ensuring fairness to all users, QoS provisioning, reducing complexity etc. A good scheduler should be capable of exploiting fast variations in channel conditions, besides maintaining fairness between the users. The proposed scheduler aims at uniform distribution of resources among users to ensure fairness, and at the same time maximize the overall system capacity within a cell.

\section{The Proposed Downlink Scheduling MECHANISM}

In this section, a new algorithm New-SCH Algorithm for DL scheduling is proposed, which is based on the method proposed in [33]. The new algorithm makes use of Assignment Model of Operations research [38] for UEs to RBs mapping during each TTI. This technique improves the overall throughput performance of the cell, while maintaining fairness in the resource allocation among the users. The details of New-SCH Algorithm are given in Algorithm 1, which outlines the steps involved in DL scheduling during each TTI.

The algorithm presumes that each eNodeB gets channel feedback information in the form of CQIfeedback Matrix, of the size equal to (No. of UEs x No. of RBs in a TTI), for all TTIs. The proposed algorithm in time-domain selects UEs to be scheduled during each TTI taking into consideration about fairness. In frequencydomain, it maps UEs to appropriate RBs, such that the net throughput performance of the cell is optimized, besides maintaining fairness. The Assignment Model used in this algorithm maps UEs to RBs during each TTI, such that the net cost of the assignment in CQI-feedback Matrix is maximized. This enables improvement in throughput performance, besides maintaining fairness.

Algorithm 1. New-SCH Algorithm

1. Obtain the Channel information for different UEs in the form of CQI-Feedback Matrix, for current TTI.

2. Choose the users to be scheduled during current TTI, giving preference to those users which were less scheduled and not scheduled in previous TTIs.

3. Map Users to RBs during current TTI, using Assignment Model so that the cost of allocation in CQI-Feedback Matrix is maximized.

4. Schedule the Users during the current TTI.

5. Repeat the above steps for each TTI. 


\section{RESULTS AND DISCUSSIONS}

\section{A. Simulation Parameters}

The implementation of the new scheduling algorithm, New-SCH Algorithm is done using a MATLAB based LTE link level simulator from the Vienna University [36], that implements standard compliant LTE downlink. It can carry out simulations in single-downlink, single-cell, multi-user and multi-cell scenarios. The simulator enables emulation of all the transmission features between Base station and UEs.

The simulations are carried out for frequency-selective channels modeled by ITU for Pedestrian-B (Ped-B) channels. Simulations are performed for 6, 15 and 25 users, choosing the bandwidths of $1.4,3$ and $5 \mathrm{MHz}$ respectively. A single cell, multi-user scenario is chosen for simulation. The simulation parameters used are listed in Table II.

Table II. Simulation Parameters

\begin{tabular}{|c|c|}
\hline Parameter & Value \\
\hline Number of Base stations & 1 \\
\hline Channel type & Pedestrian-B \\
\hline & Best CQI, \\
Scheduling Algorithms & Round-Robin, \\
& New-SCH Algorithm, \\
& MS-Algorithm \\
\hline Number of UEs & $6,15,25$ \\
\hline Bandwidth (in MHz) & $1.4,3,5 \mathrm{MHz}$ \\
\hline Simulation length & 100 Subframes \\
\hline Transmission Mode & SISO \\
\hline
\end{tabular}

\section{B. Simulation Results and Discussions}

The performance evaluation of the proposed scheduling algorithm based on simulation results is explained in this section. The performance is measured in terms of the overall cell throughput and Block Error ratio (BLER). The simulation results of the New-SCH Algorithm are compared with Round Robin, Best CQI and MY_SCH-algorithms (or MS Algo) proposed in [33]. The performance analysis of individual users in terms of individual user throughput is done to study distribution of

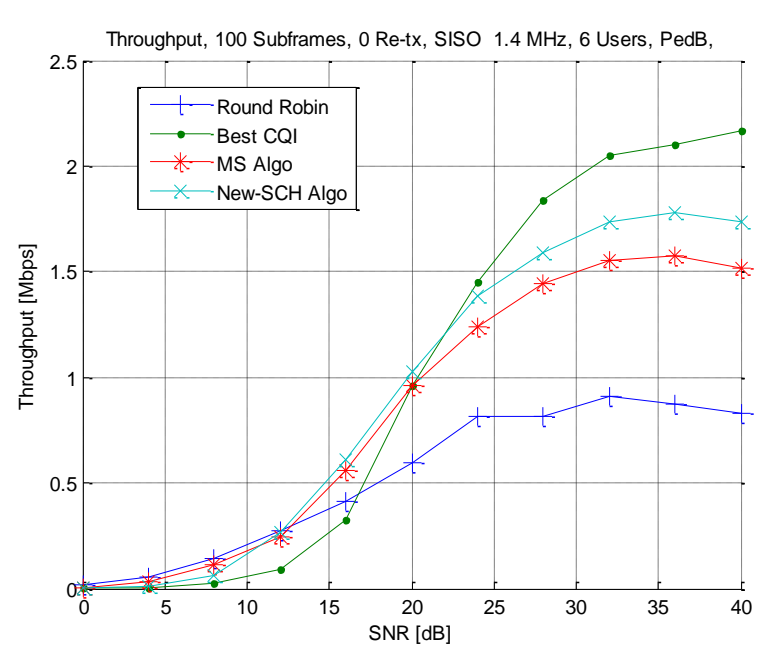

(a)

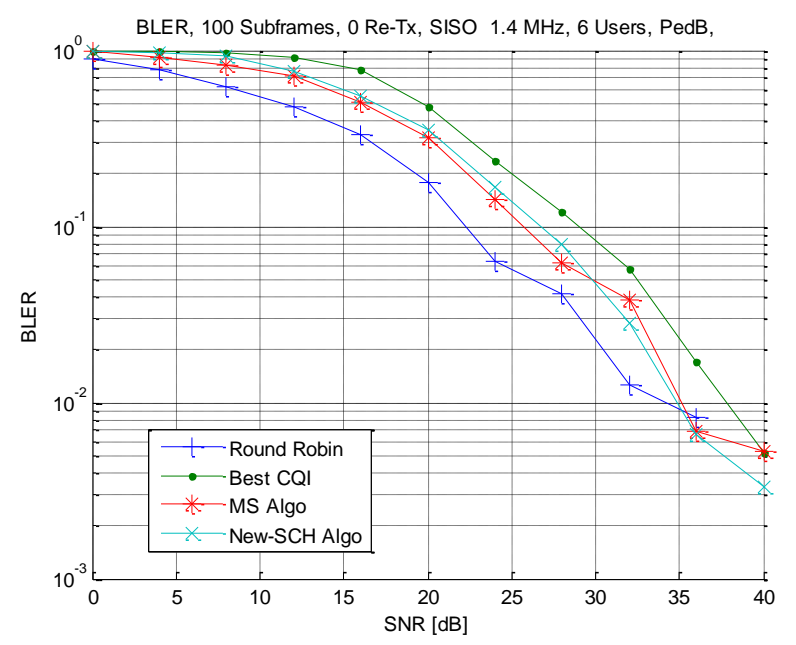

(b)

Figure 4: (a) Cell throughput (b) BLER for 6 users, BW= 1.4 $\mathrm{MHz}$

radio resources among users in different scheduling techniques.

The overall cell throughput and BLER plots for different conditions: 6 users at $1.4 \mathrm{MHz}, 15$ users at 3 $\mathrm{MHz}$ and 25 users at $5 \mathrm{MHz}$ bandwidths (BW) are shown in Fig. 4, 5 and 6 respectively. In all the cases, it is observed that the New-SCH Algorithm shows good throughput performance closer to that of the Best-CQI, compared to other algorithms. There has been the improvement in the throughput in case of $\mathrm{New}-\mathrm{SCH}$ algorithm, without costing much in BLER performance, at different SNRs.

The throughput performances of 6 users operating with selected bandwidth of $1.4 \mathrm{MHz}$, at SNRs ranging between 0-40 dBs, in different scheduling algorithms is shown in Fig. 7. While the Best-CQI method offers best throughput performance, it is least in case of Round Robin method. The fairness in the distribution of resources among all the UEs, by each of the scheduling algorithm can be observed by the amount of variations in users throughput performances at different SNRs. The throughput fluctuations are more among users in case of Best CQI method, which indicates that it is less fair in the 


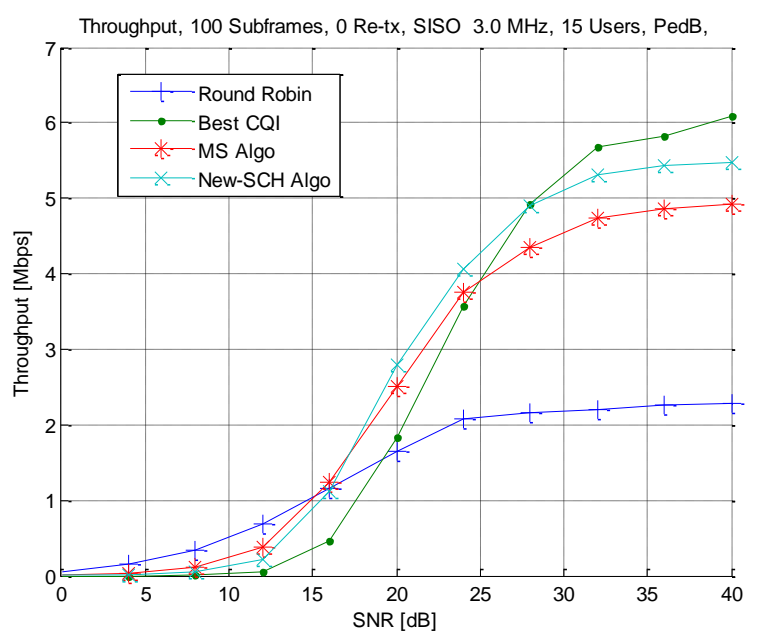

(a)

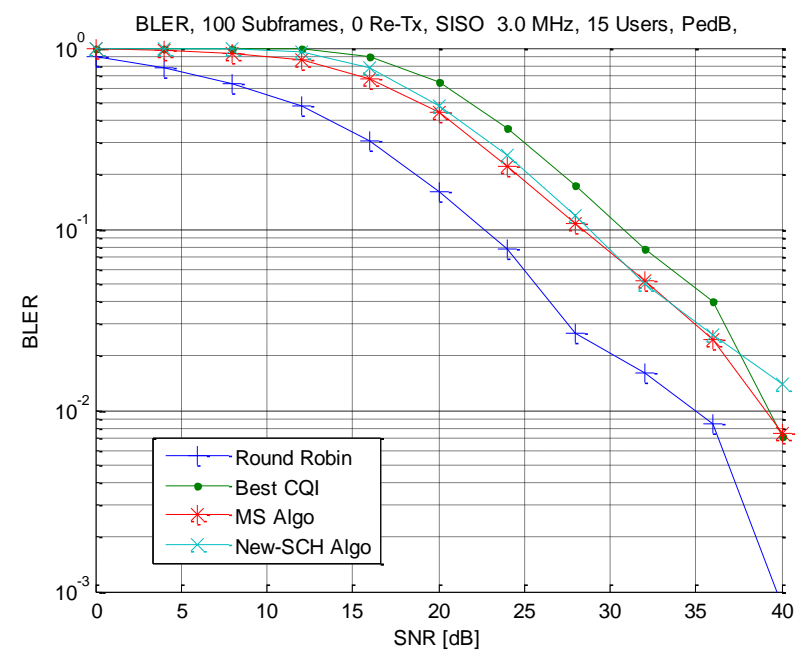

(b)

Figure 5: (a) Cell throughput (b) BLER for 15 users, $\mathrm{BW}=3.0 \mathrm{MHz}$

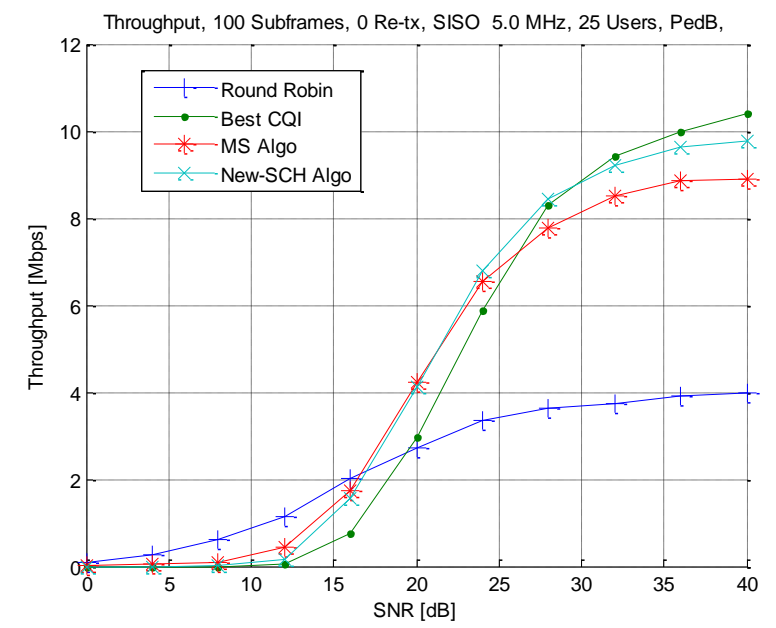

(a)

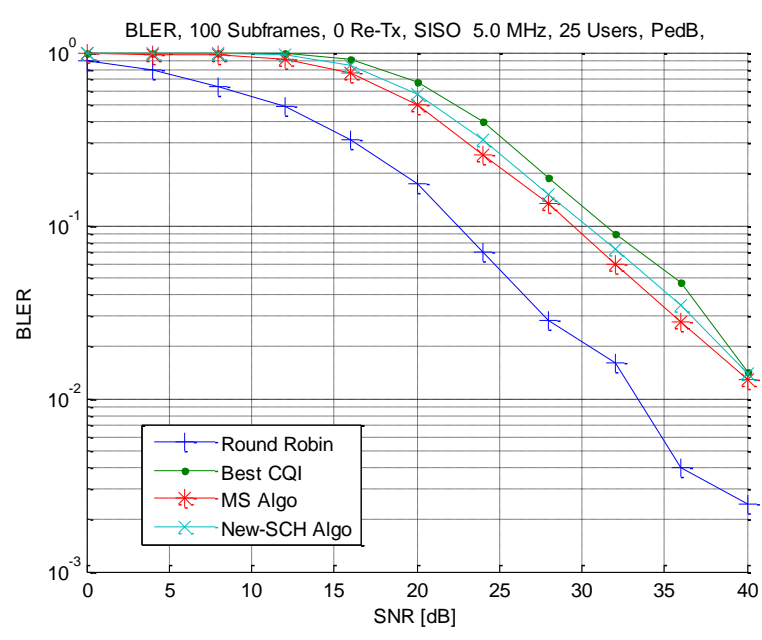

(b)

Figure 6: (a) Cell throughput (b) BLER for 25 users, $\mathrm{BW}=5.0 \mathrm{MHz}$

distribution of resources among UEs. The lesser variations among the throughputs of different users, in case of MS-Algorithm and New-SCH algorithm indicates better fairness in the allocation of resources. It is seen from the plots that New-SCH algorithm provides an optimal performance in terms of throughput and fairness, when compared with other scheduling techniques.

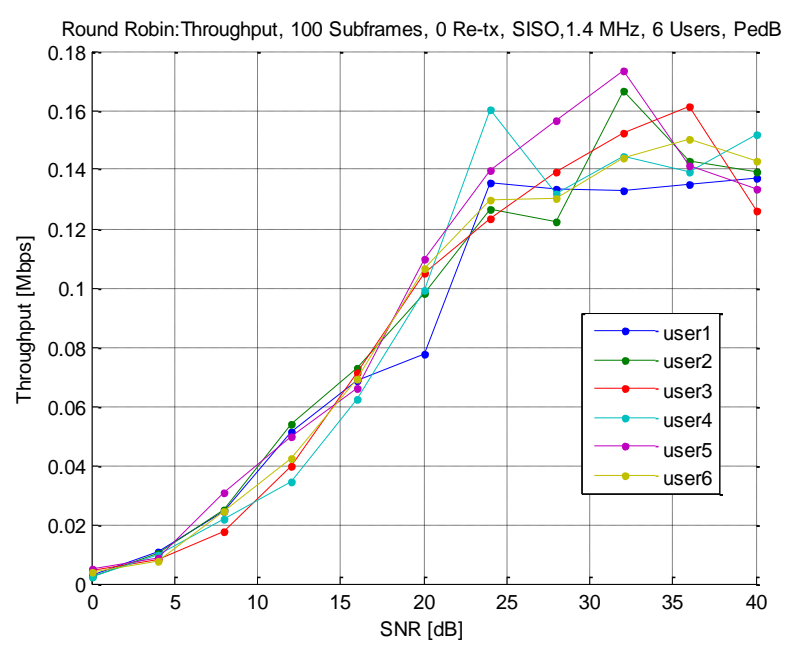

(a) 


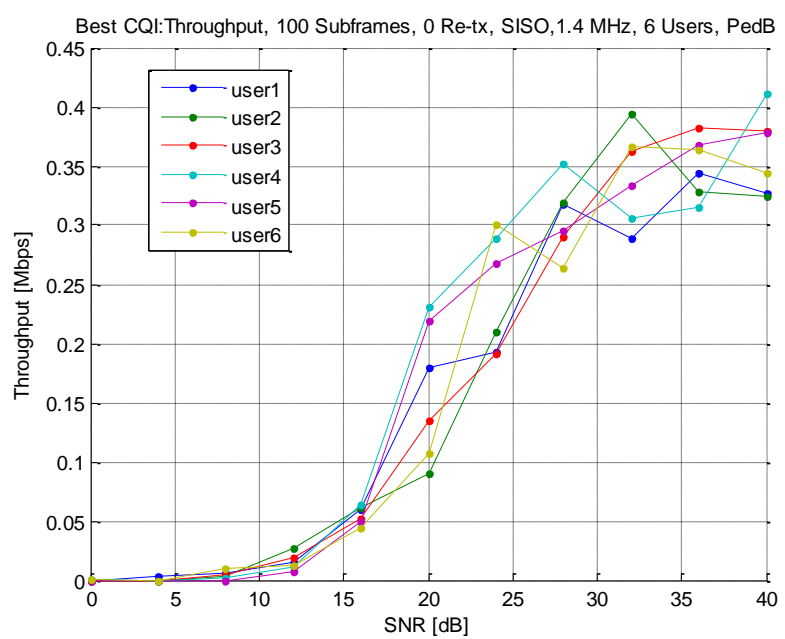

(b)

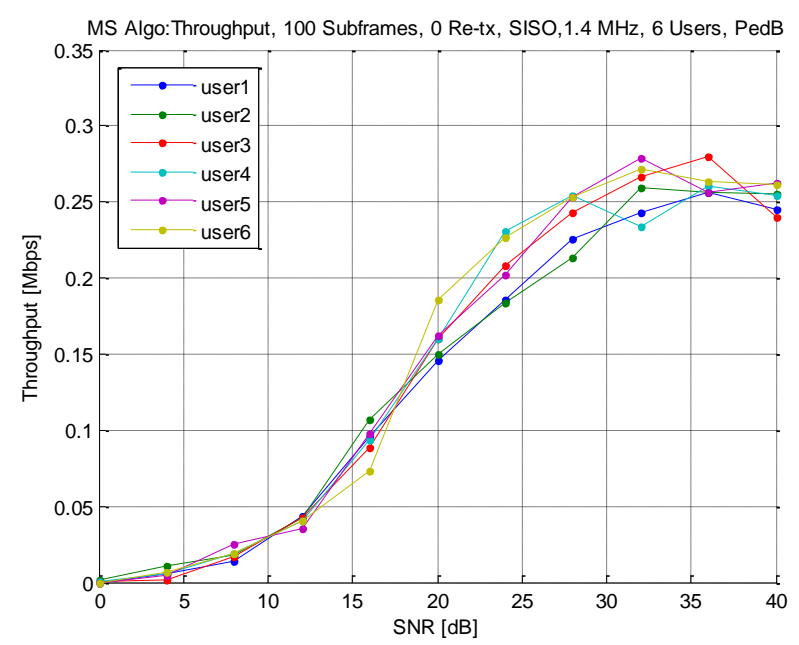

(c)

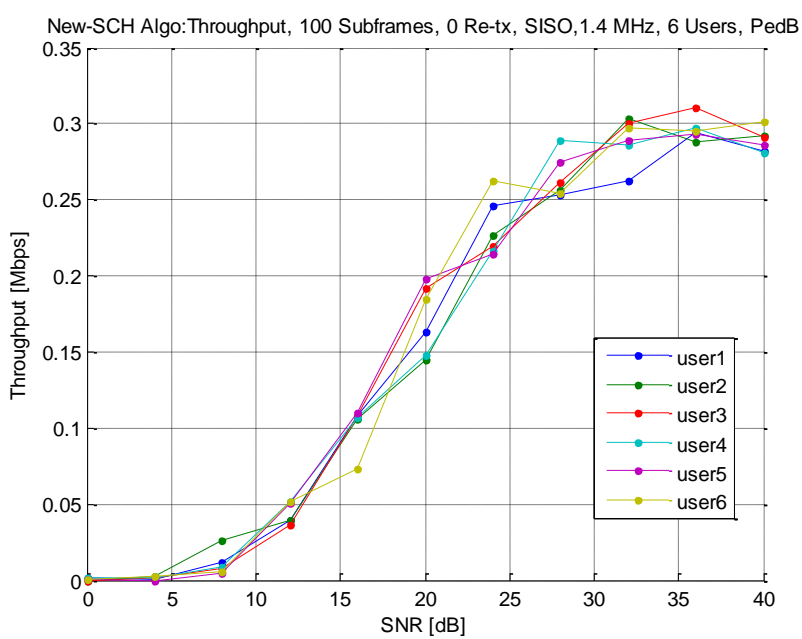

(d)

Figure 7: User througputs for (a) Round Robin (b) Best CQI (c) MS-Algorithm (d)New-SCH Algoritm

The bar graph of Fig. 8 shows the individual throughput performances of 6 users operating with 1.4 $\mathrm{MHz}$ bandwidth, at a constant SNR of $40 \mathrm{~dB}$. The figure shows that the New-SCH Algorithm provides userthroughput performance closer to that of Best CQI method compared to other scheduling techniques. The

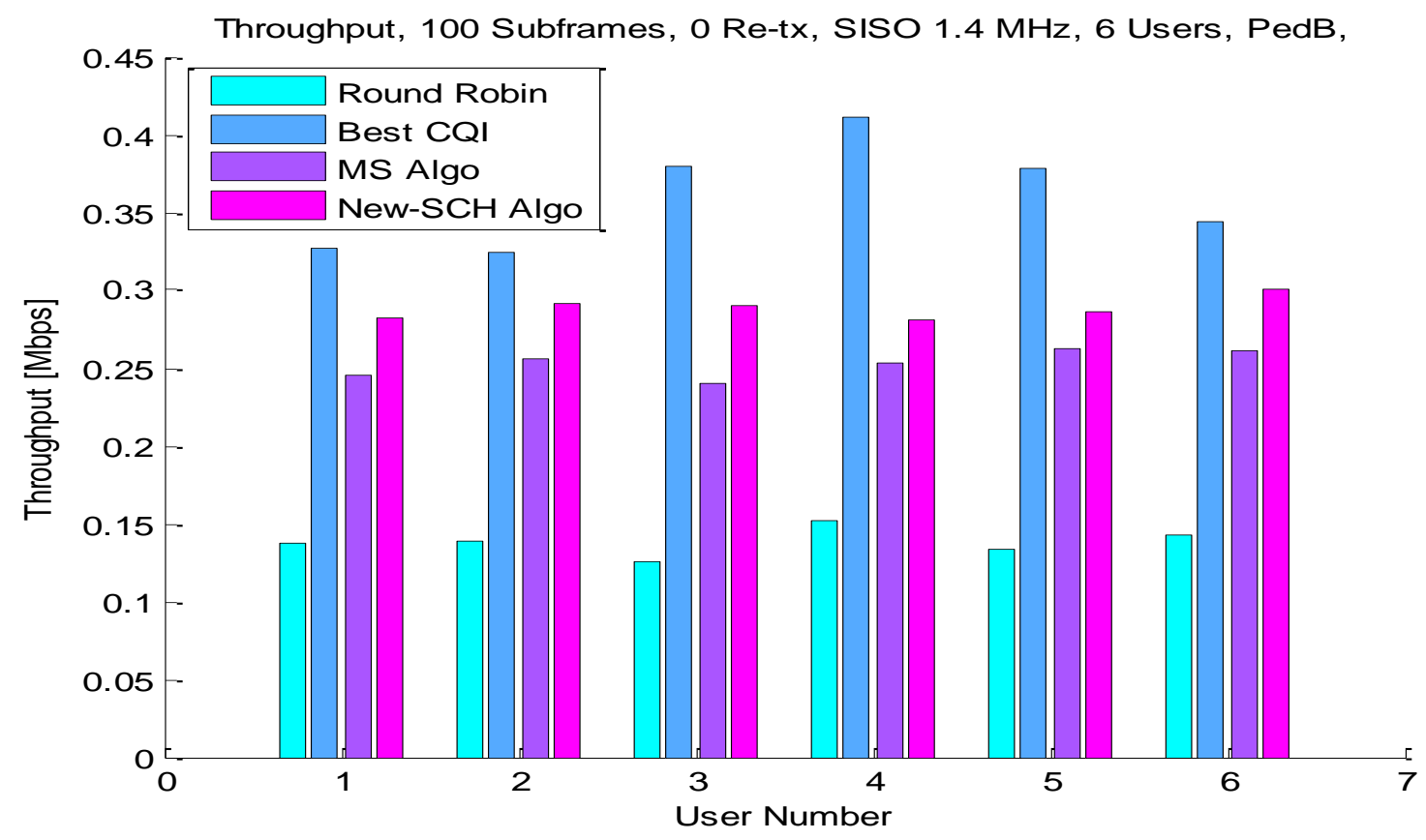

Figure 8: Throughputs of different users for different scheduling algorithms at SNR=40. 
lesser fluctuation in the throughputs among users in New$\mathrm{SCH}$ Algorithm is indicative of its ability to distribute resources to all users in a fair manner.

\section{CONCLUSIONS}

3GPP LTE network is intended to deliver high speed data and multimedia services to next generation mobile users. LTE downlink scheduling, an entity of RRM, plays a vital role in the allocation of shared radio resources among UEs. The scheduler needs to address the tradeoff that exists between throughput performance and fairness among users. This paper proposed a novel DL scheduling algorithm using Assignment Model, which effectively maps UEs to RBs during each TTI. The proposed algorithm is simulated and the results are compared with three different scheduling algorithms available in literature. The performance of the new algorithm is investigated in terms of throughput and BLER to analyze its performance at both cell-level and user level. The simulator results show that the new algorithm balances well between throughput and fairness.

\section{REFERENCES}

[1] E. Dahlman, S. Parkvall, J. Skold, P. Beming, "3G Evolution: HSPA and and LTE for Mobile Broadband", Academic Press, 2007.

[2] S. Sesia, I. Toufik, and M. Baker, "LTE - The UMTS Long Term Evolution From Theory to Practice”, John Wiley \& Sons Ltd., 2009.

[3] H. Ekstrom et al., "Technical Solutions for the $3 \mathrm{G}$ Long-Term Evolution", IEEE Communication Magazine, vol.44, no.3, pp.38-45, March 2006.

[4] Agilent, "3GPP Long Term Evolution: System Overview, Product Development and Test challenges", Application Note, 2008.

[5] Astely et al., "LTE: The Evolution of Mobile broadband", IEEE Communication Magazine, vol.47, no.4, p.44-51, April 2009..

[6] S. M. Chadchan, C. B. Akki, "3GPP LTE/SAE: An Overview", International Journal of Computer and Electrical Engineering, vol.5, no.2, pp.806-814, Oct.2010.

[7] 3GPP TR 25.913, Requirements for EUTRA and EUTRAN, v9.0.0 (2009-12).

[8] 3GPP TR 36.913, Requirements for further advancements for E-UTRA, v.9.0.0, (2009-12).

[9] R. Van Nee, R. Prasad, "OFDM for Wireless Multimedia Communications", Artech House Publications, 2000.

[10]H. Holma, A. Toskala, "LTE for UMTS-OFDMA and SC-FDMA Based Radio Access", John Wiley \& Sons Ltd., 2009.

[11] G. L. Stuber et al., "Broadband MIMO-OFDM Wireless commn", Proceedings of IEEE, vol.92, no.2, pp.271-294, Feb.2004.
[12] S. M. Chadchan, C. B. Akki, "Quality of Service Provisioning in 3GPP EPS", Journal of Telecommn. Management, vol.3, no.1, pp.36-50, April 2010.

[13] H. Ekstrom, "QoS Control in the 3GPP Evolved Packet System", IEEE Communication Magazine, vol.47, no.2, pp.76-83, Feb. 2009.

[14] R. Ludwig, H. Ekstrom, P. Williars, N. Lundin, “An Evolved 3GPP QoS Concept", In Proc. IEEE VTC, Spring 2006.

[15] S. M. Chadchan, C. B. Akki, "The Survey of Handoff Issues in Wireless ATM Networks", International Journal of Nonlinear Science, vol.7, no.2, pp.189-200, April 2009.

[16] 3GPP TS 23.107, Quality of Service (QoS) concept and Architecture, v8.0.0, (2008-12).

[17] 3GPP TS 23.207, End-to-end Quality of Service (QoS) concept and Architecture, v8.0.0, (2008-12).

[18]3GPP TS 36.133, Evolved Universal Terrestrial Radio Access (E-UTRA) Requirements for support of radio resource management, v8.1.0, (2008-03).

[19] 3GPP TS 36.321, EUTRA; Medium Access Control (MAC) protocol specification (Rel.11), v.11.0.0.(2012-09).

[20] Roke Manor Research, "LTE MAC Scheduler \& Radio Resource Scheduling", 2011.

[21] Roke Manor Research, "LTE eNodeB MAC Scheduler Interface", 2009.

[22] A. Larmo, M. Lindström, M. Meyer, G. Pelletier, J. Torsner, H. Wiemann, "The LTE Link-Layer Design”, IEEE Communications magazine, 2009.

[23] M. Gidlund, J-C Laneri, "Scheduling Algorithms for 3GPP Long-Term Evolution Systems: From a Quality of Service Perspective”, IEEE Communications magazine, 2008.

[24] A. Venkatesh, B. Parameshwaran, S. Chaudhuri, "Opportunistic Proportionate Fairness Adaptive Scheduler for High Speed Packet Access System”, In 2nd International Conference on Communication Systems Software and Middleware (COMSWARE), pp.1-8, January 2007.

[25] R. Kwan, C. Leung, J. Zhang, "Multiuser Scheduling on the Downlink of an LTE Cellular System", Research letters in Commn, Hindawi Publishing Corporation, May 2008.

[26] S. Lee, et al., "Proportional Fair Frequency-Domain Packet Scheduling for 3GPP LTE Uplink", INFOCOM, 2009.

[27] D. Jiang, H. Wang, E. Malkamaki, E. Tuomaala, "Principle and Performance of Semi-Persistent Scheduling for VoIP in LTE System", In IEEE International Conference in Wireless Communications, Networking and Mobile Computing (WiCom), pp.2861-2864, Sept. 2007.

[28] S. Saha, R. Quazi, "Priority-coupling-a semipersistent MAC scheduling scheme for VoIP traffic on 3G LTE", In 10th IEEE Conference on Telecommunications, pp.325-329, June 2009.

[29] G. Li and H. Liu, "Downlink Radio Resource Allocation for Multi-cell OFDMA System", IEEE 
Transactions on Wireless Communications, vol.5, no.12, pp. 3451-3459, December 2006.

[30] F. D. Calabrese et al., "Search-Tree Based Uplink Channel Aware Packet Scheduling for UTRAN LTE”, In Proc IEEE VTC Spring, pp. 1949-1953, May 2008.

[31] D. Sabella, M. Caretti, R. Fantini, "Energy efficiency evaluation of state of the art packet scheduling algorithms for LTE", In Proc. of IEEE European Wireless Conference, pp.1-4, April 2011.

[32] S. Videv, H. Haas, "Energy-efficient scheduling and bandwidth-energy efficiency trade-off with low load", In Proc. IEEE International Conference on Comm, pp.1-5, June 2011.

[33] Talevski, Gavrilovska, "Novel Scheduling Algorithms for LTE Downlink Transmission", Telfor Journal, vol.4, no.2, pp.24-25, 2012.

[34] Zaki, Y., "Multi-QoS-Aware Fair Scheduling for LTE”, In Proc. IEEE VTC, pp.1-5, May 2011.

[35] Tran, S.V., "Optimized scheduling algorithm for LTE downlink system", Proc. IEEE Wireless Communications and Networking Conference (WCNC), April 2012.

[36] C. Mehlfuhrer, M. Wrulich, J. Colom Ikuno, D. Bosanska, and M. Rupp, "Simulating the Long Term Evolution", In Proc. of 17th EUSIPCO, Glasgow, 2009.

[37] 3GPP TS 36.101 E-UTRA User Equipment radio transmission and reception (Rel.10), v.10.8.0 (201209)

[38] Hillier, Lieberman, "Introduction to Operations research", Mc Graw Hill Publications, 2001.

S. M. Chadchan received his Bachelor degree in Electronics and Communication Engineering from P.D.A. Engineering College, Gulbarga, India in 1991 and Master degree in Digital Electronics and Advanced Communications from Karnataka Regional Engineering College, Surathkal, India in 1994. Currently, he is Associate Professor, at BLDEA's V. P. Dr. P. G. Halakatti College of Engineering and Technology, Bijapur, India. He is pursuing his research on Mobility Management in 3GPP LTE/SAE networks. His fields of interest are Computer Networks and Wireless Communications.

C. B. Akki received his Bachelor degree in Electrical Engineering from University Vishvesvaraiah College of Engineering, Bangalore, India in 1982. He has received his Master degree and Ph.D in Computer Science and Technology from University of Roorkee, India in 1990 and 1997 respectively. He is currently Senior Architect at Talent Transformation division of Wipro Technologies, Bangalore, India. He has both academic and industrial experience in India and abroad. His special interests are Wireless Communications, Mobile Computing and Computer Networks. 